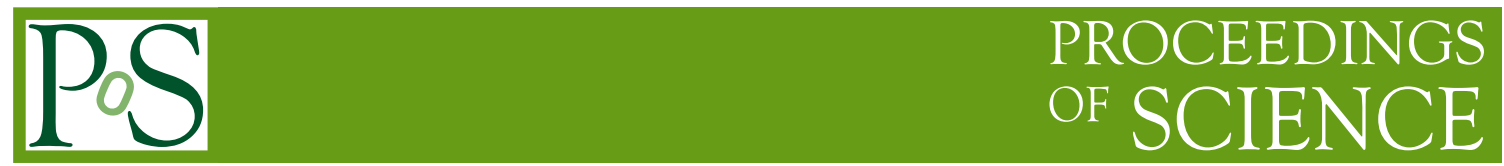

\title{
Using $K^{0} \pi^{-} \rightarrow \pi^{-}$transitions to compute $K \rightarrow(\pi \pi)_{I=0}$ decay amplitudes at NLO in the chiral expansion
}

\author{
Changhoan Kim* and Christopher Sachrajda \\ School of Physics and Astronomy \\ University of Southampton Highfield, Southampton, UK \\ E-mail: chateau@phys.soton.ac.uk, cts@phys.soton.ac.uk
}

\begin{abstract}
It is proposed to compute matrix elements for the (unphysical) $K^{0} \pi^{-} \rightarrow \pi^{-}$transition to determine the next-to-leading order low energy constants of the weak chiral Lagrangian. This allows us to evaluate $K \rightarrow(\pi \pi)_{I=0}$ decay amplitudes at this level of precision. This approach has several significant advantages over the use of $K \rightarrow \pi \pi$ transitions, most notably the elimination of $s$-channel disconnected diagrams and the use of fewer inversions.
\end{abstract}

The XXV International Symposium on Lattice Field Theory

July 30-4 August 2007

Regensburg, Germany

${ }^{*}$ Speaker. 


\section{Introduction}

Nonleptonic kaon decays present a number of major challenges. Direct $C P$ violation in kaon decays has been confirmed by the non-zero measurements of $\varepsilon^{\prime} / \varepsilon[1,2]$, but a quantitative theoretical understanding, even within the standard model, is still lacking. The $\Delta I=1 / 2$ rule also remains a longstanding puzzle. Whilst these are weak decays, the relevant energy scale is of the order of a few hundred $\mathrm{MeV}$ at which non-perturbative QCD effects are significant making the evaluation of the amplitudes difficult.

The lattice formulation of QCD together with large scale numerical simulations provide the opportunity to compute the non-perturbative effects from first principles. However, with the computing resources which are currently available it is not possible to perform the simulations directly at the physical values of $m_{u}$ and $m_{d}$. Although the situation is rapidly improving, it is still unlikely that computations with pion masses of about $140 \mathrm{MeV}$ will be performed in the near future. It is proving particularly useful in general, and for kaon decays in particular, to use chiral perturbation theory $(\chi \mathrm{PT})$ to obtain physical results from simulations with heavier values of $m_{u, d}$ [3]. In this approach, the QCD operators in the effective weak Hamiltonian are written in terms of a sum of operators composed of meson fields in the same chiral representation. A priori, the coefficients of the operators in the resulting Weak Chiral Lagrangian, i.e. the low energy constants (LECs), are unknown. The aim is to determine the LECs from the mass and momentum dependence of matrix elements computed in lattice simulations. This is well understood for $\Delta I=3 / 2 K \rightarrow \pi \pi$ decays in which the two-pion state has isospin $I=2$. In this paper we focus on $\Delta I=1 / 2$ decays in which the two pions have $I=0$

At leading order in $\chi \mathrm{PT}$, there are only a small number of LECs. For example, for the $\Delta S=1$ operator in the $(8,1)$ representation there are 2 LECs, $\alpha_{1}^{(8,1)}$ and $\alpha_{2}^{(8,1)}$, in terms of which the $K \rightarrow \pi \pi, K \rightarrow \pi$ and $K \rightarrow$ vacuum matrix elements are:

$$
\begin{aligned}
\left\langle\pi^{+} \pi^{-}\left|\mathscr{O}^{(8,1)}\right| K^{0}\right\rangle & =\frac{4 i}{f^{3}}\left(m_{K}^{2}-m_{\pi}^{2}\right) \alpha_{1}^{(8,1)}, \\
\left\langle\pi^{+}\left|\mathscr{O}^{(8,1)}\right| K^{0}\right\rangle & =\frac{4 m_{M}^{2}}{f^{2}}\left(\alpha_{1}^{(8,1)}-\alpha_{2}^{(8,1)}\right), \\
\left\langle 0\left|\mathscr{O}^{(8,1)}\right| K^{0}\right\rangle & =\frac{2 i}{f}\left(m_{K}^{2}-m_{\pi}^{2}\right) \alpha_{2}^{(8,1)}
\end{aligned}
$$

Thus by computing the $K \rightarrow \pi$ and $K \rightarrow$ vacuum matrix elements, $\alpha_{1,2}^{(8,1)}$ can be determined and the $K \rightarrow \pi \pi$ matrix elements can be evaluated at leading order. Such calculations were performed in 2001 with quenched ensembles and with meson masses above about $600 \mathrm{MeV}$ [4,5]. While these calculations demonstrated the feasibility of the procedure, it is clear that, in addition to using dynamical quarks it is necessary to perform simulations at lighter masses and to go beyond the leading order in the chiral expansion in order to understand the $\Delta I=1 / 2$ rule and the value of $\varepsilon^{\prime} / \varepsilon$. The increase in computer power since 2001 and Next-to-Leading Order (NLO) calculations in $\chi$ PT [6-10], make it possible to contemplate such an endeavour. At NLO in $\chi$ PT, the number of LECs to be determined grows and it is not possible to determine them all from $K \rightarrow \pi$ and $K \rightarrow$ vacuum matrix elements alone.

One possible approach to the determination of the NLO LECs is to compute $K \rightarrow \pi \pi$ matrix elements. However, as explained in the following section, there are difficulties in studying two- 
pion states with isospin $\mathrm{I}=0$. In this talk, we present an alternative procedure for determining all the necessary LECs at NLO, based on the evaluation of $K^{0} \pi^{-} \rightarrow \pi^{-}$transitions (see sec.3). A detailed analysis of this proposal will be presented in a paper which is currently in preparation.

\section{Difficulties in the Evaluation of $K \rightarrow(\pi \pi)_{I=0}$ Matrix Elements}

In this section we discuss the evaluation of $K \rightarrow \pi \pi$ matrix elements and the difficulty encountered by the presence of $s$-channel disconnected contractions. For illustration, consider the process $K^{0} \rightarrow \pi^{+} \pi^{-}$, studied in $\chi \mathrm{PT}$ in refs. [7,8]. Using the operator product expansion, the amplitude can be written in terms of matrix elements of the $\Delta S=1$ weak effective Hamiltonian which contains 10 four-quark operators $Q_{i}(i=1-10)$ :

$$
\left\langle\pi \pi\left|\mathscr{H}_{\Delta S=1}\right| K\right\rangle=\frac{G_{F}}{\sqrt{2}} \sum_{i=1}^{10} V_{C K M}^{i} c_{i}(\mu)\left\langle\pi \pi\left|Q_{i}\right| K\right\rangle_{\mu}
$$

where $V_{C K M}^{i}$ are appropriate combinations of CKM matrix elements, $c_{i}(\mu)$ are Wilson coefficients and $\mu$ is a renormalization scale. To demonstrate the ideas let us consider one of the operators:

$$
Q_{1}=\bar{s}_{a} \gamma_{\mu}\left(1-\gamma_{5}\right) d_{a} \bar{u}_{b} \gamma^{\mu}\left(1-\gamma_{5}\right) u_{b}
$$

The correlation function from which the $K \rightarrow \pi \pi$ transition is determined is

$$
\begin{aligned}
C_{K \rightarrow \pi \pi}\left(t_{K}, t_{O}, t_{\pi}\right) & =\left\langle 0\left|\mathscr{O}_{2 \pi}\left(t_{\pi}\right) Q_{1}\left(t_{O}\right) \mathscr{O}_{K}\left(t_{K}\right)\right| 0\right\rangle \\
& \approx\left\langle 0\left|\mathscr{O}_{2 \pi}\right| \pi \pi\right\rangle\left\langle\pi \pi\left|Q_{1}\right| K\right\rangle\left\langle K\left|\mathscr{O}_{K}\right| 0\right\rangle e^{-E_{\pi \pi}\left(t_{\pi}-t_{O}\right)} e^{-E_{K}\left(t_{O}-t_{K}\right)},
\end{aligned}
$$

where in the last line we assume that the time intervals $t_{\pi}-t_{O}$ and $t_{O}-t_{K}$ are sufficiently large and positive so that contributions from heavier states can be neglected. $\mathscr{O}_{K}$ and $\mathscr{O}_{2 \pi}$ are interpolating operators for the kaon and two-pion states respectively. By computing $C_{K \rightarrow \pi \pi}$ one can check the validity of expected asymptotic dependence on the time intervals in eq. (2.4) and extract the matrix element $\left\langle\pi \pi\left|Q_{1}\right| K\right\rangle$.

The quark flow diagrams which contribute to the correlation function are sketched in Fig.1. The number of quark propagators, and hence inversions of the Dirac operator, required to evaluate these diagrams depends on the strategy as to which of the times $t_{K}, t_{O}$ and $t_{\pi}$ are chosen for the sources. If we choose to allow $t_{\pi}$ to vary, then we have to solve the problem of inserting the quark propagator connecting $\pi^{+}$and $\pi^{-}$in diagram (A,B,C) in Fig.1. This requires as many quark propagators as the number of time slices $t_{\pi}$. If instead we allow $t_{O}$ to vary, the insertion of the loops in diagrams (A) and (C) require a large number of inversions. If we fix both $t_{O}$ and $t_{\pi}$ then we are not able to check the time behaviour of the two-pion state. Even if it were possible to perform the large number of inversions which are required to evaluate the diagrams, from experience we expect that a very large number of configurations would be required to evaluate the disconnected diagram (C). Indeed one might try evaluating the diagrams using stochastic all-to-all propagators, but this may be noisier.

We stress that the difficulties described above are technical rather than fundamental. They are nevertheless delaying the evaluation of matrix elements with two-pion states in the $\mathrm{I}=0$ channel (in the $\mathrm{I}=2$ channel only diagram (D) needs to be evaluated, which is relatively easy). 


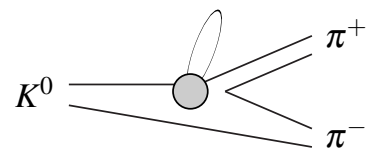

(A)

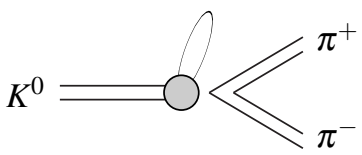

(C)

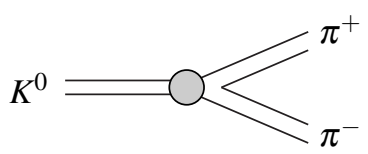

(B)

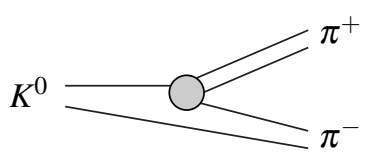

(D)

Figure 1: Quark contraction diagrams for $K \rightarrow \pi \pi$ matrix elements. The grey circle represents the insertion of a four-quark operator.

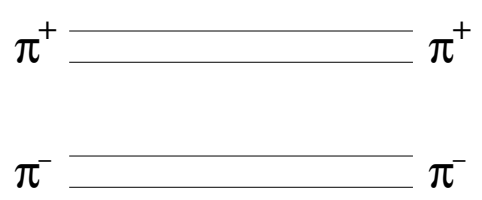

(A)

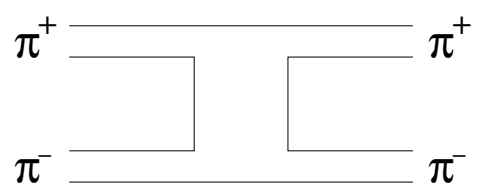

(C)

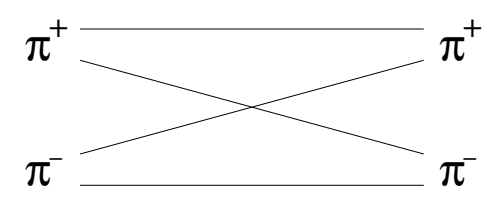

(B)
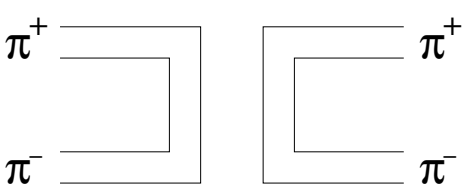

(D)

Figure 2: Quark contraction diagrams for the propagator of the $\pi^{+} \pi^{-}$state.

In order to extract the matrix element $\left\langle\pi \pi\left|Q_{1}\right| K\right\rangle$ from $C_{K \rightarrow \pi \pi}$ we need to divide by the overlap factors, including $\left\langle 0\left|\mathscr{O}_{\pi \pi}\right| \pi \pi\right\rangle$ (see eq. (2.4)). The evaluation of $\left\langle 0\left|\mathscr{O}_{\pi \pi}\right| \pi \pi\right\rangle$ requires the computation of the propagator of the $\mathrm{I}=0$ two-pion state, i.e. the evaluation of the diagrams in Fig. 2. This is at least as challenging a task as that of the $C_{K \rightarrow \pi \pi}$, with similar issues to the ones described above.

\section{3. $K^{0} \pi^{-} \rightarrow \pi^{-}$Matrix Elements}

We start by stressing that our aim in this paper is limited to the determination of the LECs at NLO in the chiral expansion, from which we evaluate the $K \rightarrow \pi \pi$ decay amplitudes at the same level of precision. The key point is that it is possible to compute the LECs using any appropriate external states; in particular it is not necessary to use two-pion states with $I=0$. Our proposal is to determine the LECs for $\Delta S=1 \Delta I=1 / 2$ operators by computing matrix elements for the 


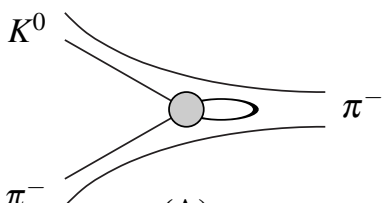

(A)

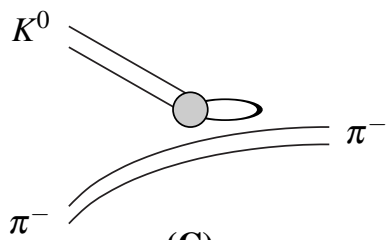

(C)

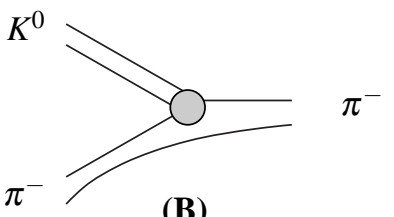

(B)

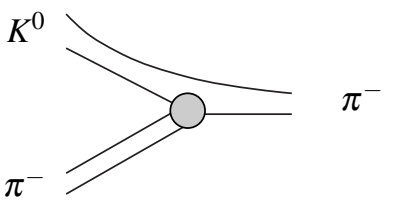

(D)

Figure 3: Quark contraction diagrams for $K \pi \rightarrow \pi$ transitions. The grey circle represents the insertion of a four-quark operator.

(unphysical) transition $K^{0} \pi^{-} \rightarrow \pi^{-}$as well as $K \rightarrow \pi$ and $K \rightarrow$ vacuum matrix elements. In this section we explain the advantages of this proposal; a detailed demonstration that all the necessary LECs can be determined from these transitions for a reasonable set of kinematic parameters will be presented in a forthcoming publication. $K^{0} \pi^{-}$is an $I=3 / 2$ highest weight state, and, as we shall see below, this leads to a number of important simplifications (analogous to those present when studying the $I=2 \pi \pi$ state).

The correlation function corresponding to $K \pi \rightarrow \pi$ transitions is

$$
\begin{aligned}
& C_{K^{0} \pi^{-} \rightarrow \pi^{-}}\left(t_{K}, t_{O}, t_{\pi}\right)=\left\langle 0\left|\mathscr{O}_{\pi^{+}}\left(t_{\pi}\right) Q_{1}\left(t_{O}\right) \mathscr{O}_{\pi^{-}}\left(t_{K}\right) \mathscr{O}_{K}\left(t_{K}\right)\right| 0\right\rangle \\
& \quad \approx\left\langle 0\left|\mathscr{O}_{\pi^{+}}\right| \pi^{-}\right\rangle\left\langle\pi^{-}\left|Q_{1}\right| K^{0} \pi^{-}\right\rangle\left\langle K^{0} \pi^{-}\left|\mathscr{O}_{K} \mathscr{O}_{\pi^{-}}\right| 0\right\rangle e^{-E_{\pi}\left(t_{\pi}-t_{O}\right)} e^{-E_{K \pi}\left(t_{o}-t_{K}\right)},
\end{aligned}
$$

where $\mathscr{O}_{\pi^{-}}$and $\mathscr{O}_{\pi^{+}}$are interpolating operators which can create or annihilate a $\pi^{-}$respectively. The quark contraction diagrams corresponding to this process are shown in Fig.3; they have common topologies with those in Fig.1. The difference is that the final-state $\pi^{+}$in Fig.1 is now crossed into the initial state where it is a $\pi^{-}$. Thus, the quark line connecting $\pi^{+}$to $\pi^{-}$in diagrams (A,B,C) of Fig. 1 now connects $\pi^{-}$to $\pi^{-}$propagating from $t_{K}$ to $t_{\pi}$ in Fig.3. We can therefore vary $t_{K}$ using a small number of quark propagators, and hence check the asymptotic exponential behavior of the $K \pi$ state.

What is more important is the absence of diagram such as (C) in Fig.1. The s-channel disconnected diagram in Fig.1(C) now becomes the $t$-channel disconnected diagram in Fig.3(C). Such $t$-channel disconnected contributions appear, for example, when studying $K \rightarrow \pi \pi$ matrix elements with an $I=2 \pi \pi$ state. There have been several calculations of $I=2 \pi \pi$ states [11,12] and this did not pose any problem.

A related advantage is that one can avoid the vacuum subtraction. For an $I=0$ final state at rest, there is mixing with the vacuum state which must be subtracted ${ }^{1}$. In this case, the correlation

\footnotetext{
${ }^{1}$ One can avoid the vacuum subtraction by considering the $\pi \pi$ state at non-zero momentum
} 


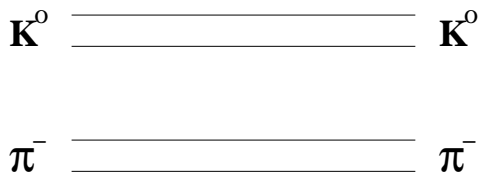

(A)

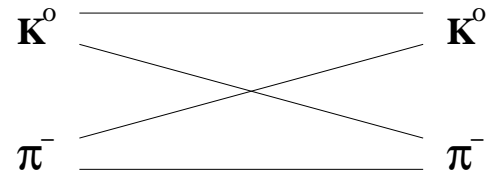

(B)

Figure 4: Quark contraction diagrams for the propagator of the $K^{0} \pi^{-}$state.

function in eq. (2.3) contains the vacuum contribution,

$$
\left\langle 0\left|\mathscr{O}_{2 \pi}\right| 0\right\rangle\left\langle 0\left|Q_{1}\right| K^{0}\right\rangle\left\langle K^{0}\left|\mathscr{O}_{K}\right| 0\right\rangle e^{-E_{K}\left(t_{O}-t_{K}\right)}
$$

which must be subtracted in order to get Eq.(2.4). There is no such subtraction necessary in the $K \pi \rightarrow \pi$ correlation function.

Note also that the overlap factor $\left\langle K^{0} \pi^{-}\left|\mathscr{O}_{K} \mathscr{O}_{\pi^{-}}\right| 0\right\rangle$ can be calculated much more easily. Fig.4 shows the quark contraction diagrams. These are the same diagrams which appear in the $I=2 \pi \pi$ propagator which is calculated without any difficulty.

When evaluating $K^{0} \pi^{-} \rightarrow \pi^{-}$matrix elements, we need to decompose the operators $Q_{i}$ into their $\Delta I=1 / 2$ and $\Delta I=3 / 2$ components. In $K \rightarrow \pi \pi$ transitions, the isospin transfer, $\Delta I$ is determined by isospin of the final state, $\Delta I=1 / 2$ if $I_{\pi \pi}=0$ and $\Delta I=3 / 2$ if $I_{\pi \pi}=2$. For the $K^{0} \pi^{-} \rightarrow \pi^{-}$ transition, the isospin of the external states is already fixed, $I_{K^{0} \pi^{-}}=3 / 2, I_{\pi^{-}}=1$, both $\Delta I=1 / 2$ and $\Delta I=3 / 2$ transitions are allowed and therefore a basis of operators with fixed isospin should be used (see for example Appendix A of ref. [5]).

Since, for both $K \rightarrow \pi \pi$ and $K \pi \rightarrow \pi$ transitions we have a two particle state, the finite-volume effects are not exponentially small and should be taken into account [14]. Just as for the $(\pi \pi)_{I=2}$ state, the lowest energy $K^{0} \pi^{-}$state(s) can be isolated and the standard techniques [14-16] can be applied. Energy, of course, must be injected at the operator (which is also the case in practice in $K \rightarrow \pi \pi$ transitions).

The observation that the initial $K^{0} \pi^{-}$state is one of highest weight also means that one can also envisage performing the $K \pi \rightarrow \pi$ calculations in the (non-unitary) partially quenched QCD, which is not the case for the standard extraction of $\Delta I=1 / 2 K \rightarrow \pi \pi$ matrix elements [13]. One might also try to improve the precision of the determination of the LECs by extending the kinematical reach of the calculations using partial twisting, which again is not possible for $K \rightarrow(\pi \pi)_{I=0}$ transitions [17, $18]$.

\section{Concluding Remarks}

In this paper we have presented a suggestion for the determination of the LECs necessary for the evaluation of the $K \rightarrow(\pi \pi)_{I=0}$ decay amplitudes at NLO in the chiral expansion. Our approach is based on the computation of $K^{0} \pi^{-} \rightarrow \pi^{-}$matrix elements (combined with $K \rightarrow \pi$ and $K \rightarrow$ vacuum matrix elements). We will demonstrate explicitly that all the LECs can be determined in this way in a forthcoming paper. We then have to develop effective strategies for implementing these ideas in numerical simulations and to investigate how precisely the LECs can be determined. 
In spite of the relative simplicity of our approach, there will be technical issues in its implementation; including the subtraction of power divergences. As is well known, some of the $\Delta S=1$ four-quark operators mix with lower dimensional ones, leading to ultra-violet divergences proportional to inverse powers of the lattice spacing. The numerical subtraction of the divergences at leading order in the chiral expansion was performed in the quenched studies of refs. $[4,5]$ and it remains to be seen how accurately this can be done in dynamical simulations at NLO in the chiral expansion.

\section{References}

[1] J. R. Batley et al. [NA48 Collaboration], Phys. Lett. B 544, 97 (2002) [arXiv:hep-ex/0208009].

[2] A. Alavi-Harati et al. [KTeV Collaboration], Phys. Rev. D 67, 012005 (2003) [Erratum-ibid. D 70, 079904 (2004)] [arXiv:hep-ex/0208007].

[3] C. W. Bernard, T. Draper, A. Soni, H. D. Politzer and M. B. Wise, Phys. Rev. D 32, 2343 (1985).

[4] T. Blum et al. [RBC Collaboration], Phys. Rev. D 68, 114506 (2003) [arXiv:hep-lat/0110075].

[5] J. I. Noaki et al. [CP-PACS Collaboration], Phys. Rev. D 68, 014501 (2003) [arXiv:hep-lat/0108013].

[6] J. Kambor, J. Missimer and D. Wyler, Nucl. Phys. B 346, 17 (1990).

[7] J. Laiho and A. Soni, Phys. Rev. D 65, 114020 (2002) [arXiv:hep-ph/0203106].

[8] J. Laiho and A. Soni, Phys. Rev. D 71, 014021 (2005) [arXiv:hep-lat/0306035].

[9] J. Bijnens, E. Pallante and J. Prades, Nucl. Phys. B 521, 305 (1998) [arXiv:hep-ph/9801326].

[10] C. J. D. Lin, G. Martinelli, E. Pallante, C. T. Sachrajda and G. Villadoro, Nucl. Phys. B 650, 301 (2003) [arXiv:hep-lat/0208007].

[11] C. Kim, UMI-31-47246, 2004

[12] T. Yamazaki et al. [CP-PACS Collaboration], Phys. Rev. D 70, 074513 (2004) [arXiv:hep-lat/0402025].

[13] C. J. D. Lin, G. Martinelli, E. Pallante, C. T. Sachrajda and G. Villadoro, Phys. Lett. B 553, 229 (2003) [arXiv:hep-lat/0211043].

[14] L. Lellouch and M. Luscher, Commun. Math. Phys. 219, 31 (2001) [arXiv:hep-lat/0003023].

[15] C. h. Kim, C. T. Sachrajda and S. R. Sharpe, Nucl. Phys. B 727, 218 (2005) [arXiv:hep-lat/0507006].

[16] N. H. Christ, C. Kim and T. Yamazaki, Phys. Rev. D 72, 114506 (2005) [arXiv:hep-lat/0507009].

[17] C. T. Sachrajda and G. Villadoro, Phys. Lett. B 609 (2005) 73 [arXiv:hep-lat/0411033].

[18] P. F. Bedaque and J. W. Chen, Phys. Lett. B 616 (2005) 208 [arXiv:hep-lat/0412023]. 